\title{
El concepto de serie numérica. Un estudio a través del modelo de Pirie y Kieren centrado en el mecanismo "folding back"
}

\author{
M. Laura Delgado Martín, Universidad de Salamanca (España) \\ Myriam Codes Valcarce, Universidad de Salamanca (España) \\ M. Consuelo Monterrubio Pérez, Universidad de Salamanca (España) \\ M. Teresa González Astudillo, Universidad de Salamanca (España)
}

Recibido el 1 de Marzo de 2014; aceptado el 3 Octubre de 2014

El concepto de serie numérica. Un estudio a través del modelo de Pirie y Kieren centrado en el mecanismo "folding back"

\section{Resumen}

En este artículo se presenta una investigación cuyo objetivo es caracterizar el proceso que sigue un grupo de alumnos universitarios para construir una serie numérica y determinar su convergencia. Para ello se analiza la actividad de dichos alumnos cuando resuelven una tarea en el aula habitual siguiendo el modelo propuesto por Pirie y Kieren. Esto ha permitido describir su progresión a través de los diferentes niveles de comprensión y comprobar la necesidad, en determinadas ocasiones, de realizar una vuelta a niveles inferiores mediante el mecanismo "folding back". Se han encontrado manifestaciones del folding back de diferente naturaleza.

Palabras clave: Serie numérica, comprensión, Pirie y Kieren, folding back.

O conceito de série numérica. Um estudo através do modelo de Pirie e Kieren focado no mecanismo "folding back"

\section{Resumo}

Neste artigo apresenta-se uma investigação cujo objetivo é caracterizar o processo que segue um grupo de alunos universitários para construir uma série numérica e determinar a sua convergência. Para tal analisa-se a atividade dos referidos alunos quando resolvem a tarefa na aula habitual seguindo o modelo proposto por Pirie e Kieren. Isto permitiu descrever a sua progressão através dos diferentes níveis de compreensão e comprovar a necessidade, em determinadas ocasiões, de realizar uma volta a niveis inferiores mediante o mecanismo "folding back". Encontraram-se manifestações do folding back de diferente natureza.

Palavras chave: série numérica, compreensão, Pirie e Kieren, folding back.

Para citar: Delgado, M. L., Codes, M., Monterrubio, M. C., \& González Astudillo, M. T. (2014). El concepto de serie numérica. Un estudio a través del modelo de Pirie y Kieren centrado en el mecanismo “folding back". Avances de Investigación en Educación Matemática, 6, 25 - 44. 
The concept of numerical series. A study with Pirie and Kieren model focused on "folding back" mechanism

\begin{abstract}
In this paper we present a research about the process followed by group of university students to build a numerical series and to determine its convergence. To this aim, the activity of the students is analyzed through the Pirie and Kieren model, while they are solving a task in their usual classroom. This theoretical frame can describe their progression through the different levels of the model. We have been able to prove in several situations that it is necessary to go back to inner levels using a mechanism called "folding back", which can be caused by different reasons.
\end{abstract}

Key words: numerical series, understanding, Pirie and Kieren, folding back

Le concept de la série numérique. Une étude suivant le modèle de Pirie et Kieren, axé sur le mécanisme "folding back"

\title{
Résumé
}

On présente dans cet article une recherché dont l'objectif c'est de caractériser le procès suivi par un groupe d'étudiants universitaires pour construire une série numérique et de déterminer sa convergence. Pour cela on analyse l'activité de ces étudiants en train de résoudre un travail dans leur classe habituelle, selon le modèle propos par Pirie et Kieren. Cela a permis de décrire leur progression à travers les différents niveaux de compréhension et vérifier la nécessité, dans certaines occasions, d'effectuer un retour à des niveaux inferieures grâce au mécanisme "folding back". On a trouvé des exemples de "folding back" de diffèrent nature.

Paroles clés: série numérique, compréhension, Pirie et Kieren, folding back.

\section{Introducción}

Las series numéricas aparecen en el currículo del primer curso de estudios superiores principalmente en los de ciencias puras e ingeniería aunque también en algunos de ciencias sociales y económicas. La relevancia de este tópico está vinculada a su aplicación a otros temas de la matemática superior como el desarrollo en series de Fourier, los desarrollos en series de potencias o el cálculo integral, por nombrar algunos. Además, en él confluyen tres conceptos fundamentales del Análisis Matemático: función, límite e infinito.

El concepto de serie numérica ha adquirido en los últimos años un interés creciente en las investigaciones educativas tanto a nivel nacional como internacional. Prueba de ello son los trabajos que en los últimos años se han desarrollado en el seno del Grupo de Investigación de Didáctica del Análisis Matemático (GIDAM) de la Sociedad Española de Investigación en Educación Matemática (SEIEM) (Codes, 2010; Codes, Delgado, González Astudillo, \& Monterrubio, 2013; Codes, González, Delgado, \& Monterrubio, 2013; Delgado, González, Monterrubio, \& Codes, 2013) y los que se han llevado a cabo por otros grupos internacionales (Carlsen, 2010; González-Martín, Nardi, \& Biza, 2011; Martínez-Planell, González, DiCristina, \& Acevedo, 2012). Tanto la complejidad intrínseca de este concepto como la de otros que por definición están vinculados al mismo (función, límite, infinito) justifican este interés.

La mayoría de las investigaciones tratan principalmente aspectos cognitivos, concretamente la dualidad proceso/objeto de los entes matemáticos. Algunas de ellas se centran en tópicos propios del Análisis Matemático como infinito, límite o sucesiones, y han derivado resultados que son de interés para el caso particular de las 
series numéricas. Este es el caso de los trabajos de Weller, Brown, Dubinsky, McDonald, y Stenger (2004) y Dubinsky, Weller, McDonald, y Brown (2005) sobre el infinito que brindan una posible explicación de la dualidad infinito potencial-actual presente en cualquier concepto que conlleve un proceso infinito. También del trabajo de McDonald, Mathews, y Strobel (2000) sobre sucesiones numéricas han surgido algunos resultados como la importancia de establecer vínculos entre ciertos objetos mentales para construir un esquema en el sentido APOS (Codes, 2010) y las construcciones cognitivas de SEQLIST y SEQFUNC que se han adaptado al caso de las series numéricas (Martínez-Planell, et al. 2012).

En el caso más general de las series de funciones y empleando un software de cálculo simbólico, Kidron (2002) observó que los estudiantes concebían las sumas infinitas de tres formas: como una aproximación finita, es decir, como una suma con un número muy grande de sumandos pero finito; como un proceso, no como un producto y como un límite, es decir, como el resultado de un proceso infinito. En comparación con otras investigaciones, Kidron comprobó en un estudio experimental que un mayor porcentaje de estudiantes comenzaba a dar muestras de concebir una suma infinita como un límite, es decir, como un objeto. Este hecho lo justifica por la instrucción que recibieron, en la que el uso del software de cálculo simbólico les facilitó la transición de una concepción proceso a objeto al permitirles manejar el concepto de suma infinita como proceso y como objeto.

Para introducir el concepto de serie numérica a partir de la serie geométrica, Codes (2010) propone una actividad en la que el desarrollo histórico del concepto guía una serie de tareas que persiguen que los estudiantes establezcan vínculos entre los elementos matemáticos que componen este concepto, límite y sucesión de sumas parciales, y vínculos entre distintos modos de representación de dichos elementos. Por ejemplo, al dibujar en el plano cartesiano los primeros términos de una sucesión de sumas parciales a partir de su expresión simbólica como suma de los primeros términos de otra sucesión, $S_{n}=\sum_{k=0}^{n} a_{k}$, se establece un vínculo entre el modo de representación gráfico y simbólico del elemento sucesión, a la vez que se establece un vínculo entre las dos sucesiones implícitas en una serie infinita: $a_{k}$ y $S_{n}$.

Por otro lado, desde la perspectiva sociocultural de L. Vygotsky, Carlsen (2010) identificó cuatro elementos matemáticos en el proceso de apropiación del concepto de serie geométrica como herramienta cultural: i) cálculo de los elementos que generan una progresión geométrica: el primer sumando y la razón de la progresión; ii) obtención del término general de la progresión; iii) formulación de la suma infinita; iv) estudio de la convergencia para determinar el carácter convergente o no de la serie. Delgado et al. (2013) y Codes, Delgado et al. (2013) observaron un esquema similar cuando analizaban el trabajo de pequeños grupos de estudiantes mientras resolvían en clase una tarea que giraba en torno a series armónicas.

González-Martín et al. (2011) lideran otra línea de trabajo centrada en la enseñanza. En sus investigaciones han encontrado una fuerte resistencia institucional a considerar los resultados de las investigaciones que se realizan en didáctica de la Matemática para mejorar los procesos de enseñanza, tanto en el contenido de los libros de texto como en la práctica docente.

Para conocer con detalle el proceso que siguen los alumnos en la construcción del concepto de serie numérica, en este artículo se va a analizar el trabajo de un grupo de alumnos cuando resuelven en clase una tarea. El modelo de Pirie y Kieren, que se describe a continuación, nos proporciona un marco teórico para describir este proceso. 
El objetivo de esta investigación es caracterizar el proceso de construcción de serie numérica y la determinación de su convergencia utilizando este modelo.

\section{Marco Teórico}

El modelo de Pirie-Kieren nos permite observar y describir el proceso a través del cual el conocimiento matemático se crea y reorganiza. Se centra en cómo los estudiantes reflexionan acerca de su conocimiento matemático en un entorno de aprendizaje en el que cada uno realiza su interpretación personal mediante una actividad que resuelven en grupo (Pirie \& Kieren, 1992).

Desde este modelo, la comprensión se considera un proceso dinámico y activo en el que se combinan construcción y acciones concretas, las cuales implican un movimiento continuo entre diferentes maneras de pensar representadas en el modelo como unas "capas" o "niveles", entre las que se puede transitar, no sólo avanzando, sino también retornando a una capa interior, para reflexionar, o volver a trabajar acerca de comprensiones previas sobre un concepto matemático. Esta vuelta a una capa interior se denomina "folding back".

Este modelo teórico está estructurado en ocho capas o niveles de acción para describir el crecimiento de la comprensión de un estudiante sobre un concepto o un tópico matemático. Estas capas reciben los nombres: Primitive Knowing, Image Making, Image Having, Property Noticing, Formalising, Observing, Structuring e Inventising.

Los ocho niveles se representan en un diagrama en dos dimensiones en el que los círculos correspondientes a cada nivel están anidados uno dentro de otro. Es conveniente precisar que "el diagrama es sólo una aproximación gráfica al modelo en dos dimensiones con objeto de representarlo. No es el modelo en sí mismo, tiene muchos inconvenientes pero teniendo esto en mente, el diagrama es una herramienta útil” (Pirie \& Kieren, 1994, p. 67).

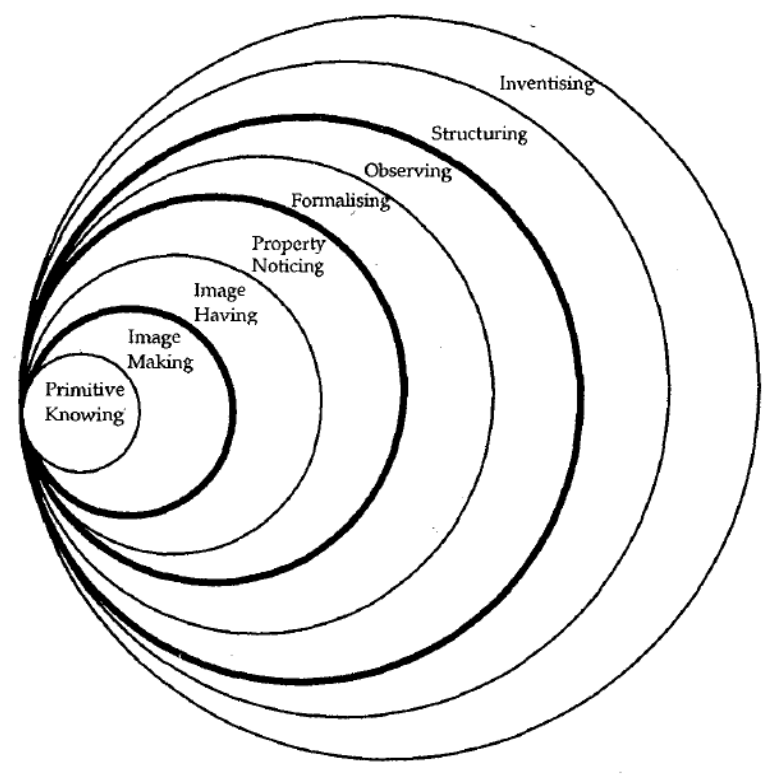

Figura 1. El modelo de Pirie-Kieren (Pirie \& Martin, 2000) 
El anidamiento de niveles, así representado, ilustra el hecho de que el crecimiento en la comprensión no es ni lineal ni unidireccional. De hecho, cada nivel contiene todos los anteriores y está incluido en los niveles siguientes. Esto enfatiza y expresa de forma gráfica el hecho de que la comprensión tiene una naturaleza "embebida", esto es, incluye dentro de sí a otros muchos (Martin, 2008).

Aunque los anillos del modelo tal y como están dibujados dan una imagen de crecimiento hacia fuera, en realidad es un movimiento continuo hacia delante y hacia atrás a través de los diferentes niveles.

Los niveles del modelo aquí descritos no deben interpretarse como una progresión de un nivel de matemática elemental a uno más avanzado o de "nivel exterior"; hacen referencia a la comprensión del conocimiento de un individuo cuando se enfrenta a una tarea matemática. A medida que un estudiante va progresando en su conocimiento mientras resuelve la tarea, su comprensión es más sólida. Puede que en la resolución de una determinada tarea no se avance significativamente, no se crucen fronteras entre niveles, todo va a depender de su complejidad y de cómo esté diseñada.

A pesar de esto, los últimos niveles del modelo tienen una naturaleza mucho más compleja y sólo se alcanzan en actividades correspondientes a matemáticas superiores, en las que los conceptos manejados permiten llegar a este grado de sofisticación.

A continuación se describen cada uno de los niveles del modelo (Pirie \& Kieren, 1994):

- Primitive Knowing. Este nivel corresponde a todo lo que el estudiante conoce y sabe hacer, excepto lo relativo al nuevo concepto matemático que se le presenta. Es importante hacer referencia a él, porque el observador (profesor y/o investigador) nunca sabe exactamente qué conoce el estudiante. En definitiva, el término Primitive Knowing no debe confundirse con un nivel bajo de matemáticas.

- Image Making. En este nivel el alumno realiza acciones ancladas en la representación particular del nuevo tópico matemático, mediante las que intenta hacerse una idea acerca de lo que trata. Estas imágenes no tienen por qué ser únicamente visuales o relacionadas con algún dibujo, y se pueden expresar mediante el lenguaje o acciones concretas.

- Image Having. En este nivel, el estudiante está liberado de acciones particulares y de ejemplos concretos y puede elaborar y llevar a cabo un plan mental sobre las acciones que debe realizar.

- Property Noticing. Este es el primer paso hacia la generalización matemática, ya que el alumno es capaz de examinar propiedades y establecer conexiones y, lo que es fundamental, es capaz de expresarlas correctamente, tanto para él mismo, como para el observador o para otro compañero. En el nivel Property Noticing el alumno es capaz de cuestionar tanto sus propias comprensiones como las de otros y busca qué puede decirse de forma general.

- Formalising. En este nivel, el estudiante es capaz de pensar conscientemente acerca de las propiedades, de sus generalizaciones y trabajar con el concepto como un objeto formal.

- Observing. Una persona que ha llegado al nivel Formalising, también está en disposición de plasmar y coordinar tal actividad formal y reflejarla en forma 
de teoremas, es entonces cuando diremos que los estudiantes se encuentran en este nivel.

- Structuring. Este nivel aparece cuando pensamos en las observaciones formales como una teoría, con una estructura interna concreta e inalterable. Esto significa que la persona es consciente de cómo un conjunto de teoremas están interrelacionados y necesitan una demostración formal o verificación, mediante argumentos meta-matemáticos o lógicos.

- Inventising. Este es el nivel exterior del modelo. En él una persona tiene una comprensión total y completa del tópico y es capaz de realizar rupturas, sobre éste $\mathrm{u}$ otros tópicos en situaciones diferentes que le permitan crear nuevas preguntas y que le lleven a otros conceptos. Es capaz de profundizar, de ir más allá en sus preguntas, de extrapolar el razonamiento y atreverse a explorar otros caminos.

Una fortaleza de esta teoría es que se centra en el camino particular de cada alumno, el que realiza para comprender un cierto concepto matemático. Este camino está mediatizado por el ambiente de aprendizaje y en él también son fundamentales las interacciones y conversaciones a través de las cuales ocurre la actividad matemática (Pirie \& Kieren, 1992).

Como se ha indicado anteriormente el tránsito a través de los niveles no es lineal ya que no se producen sólo avances sino también retrocesos. A este fenómeno los autores lo denominan "folding back" y se desencadena cuando una persona que está en un nivel de comprensión exterior del modelo, en un determinado momento puede volver a un nivel interior buscando una comprensión más intuitiva o más localizada. Cuando vuelve al nivel interior no lo hace de la misma manera que cuando inicialmente estaba en él, ya que ahora está "estimulado" y guiado por el conocimiento que ha adquirido en el nivel más exterior (Pirie \& Kieren, 1994).

Podemos pensar en el folding back a través de una metáfora puesto que lo que realmente hacemos es enriquecer, engrosar nuestra comprensión previa, por lo tanto al regresar al nivel exterior, esa comprensión es mucho más poderosa, estable y potente que la que teníamos inicialmente.

Partiendo de este planteamiento, cuando un estudiante se enfrenta a un problema desde un nivel exterior y encuentra que no es capaz de resolverlo, retorna a un nivel interior de comprensión. La actividad desarrollada en este nivel como consecuencia del folding back se hace con la perspectiva de lo que se ha adquirido en los niveles exteriores. Se está construyendo una comprensión "más gruesa" en la capa interior que luego soportará y extenderá a su comprensión de la capa exterior a la que volverá. En este proceso de reorganización el estudiante ha adquirido un grado de seguridad que le permite afrontar la tarea desde otra perspectiva.

Con este modelo se considera la compresión del conocimiento matemático en continuo cambio y se "legitimiza" la vuelta atrás a formas más simples de comprensión, a dudas razonables sobre lo que cada uno sabe y cómo lo sabe, y la absoluta necesidad de esa vuelta personal si queremos plantear un crecimiento en la comprensión de los conceptos matemáticos.

Cuando el folding back tiene lugar, el tipo de trabajo que realiza el estudiante cuando vuelve a un nivel interior del modelo puede ser de cuatro tipos (Martín, 2008): 
- Trabajo en el nivel interior usando comprensiones ya existentes, así el estudiante se involucra en tareas menos sofisticadas o menos formales matemáticamente hablando. Ocurre cuando el alumno es plenamente consciente de sus limitaciones y recupera conocimientos que le proporcionan más confianza y seguridad.

- Cuando el alumno vuelve a una capa interior a recuperar lo que sabe que necesita, hablaremos de "collecting" o "folding back to collect". Esta actividad más que cualquier acto de modificación implica una búsqueda, un encuentro y una comprensión

- A veces en un folding back el estudiante precisa abandonar el tópico en el que trabaja porque carece del conocimiento necesario para resolver la tarea retornando a la capa Primitive Knowing. Así, el engrosamiento que produce el folding back, se origina desde un área de las matemáticas diferente.

- Cuando el alumno no percibe la conexión entre sus comprensiones primitivas y la nueva en la que se está trabajando, retorna a un nivel interior comenzando de nuevo. Diremos entonces que se ha producido una discontinuidad. Esto supone construir una nueva imagen que no está relacionada con una comprensión ya existente.

Respecto al resultado del folding back, éste puede ser efectivo o no. En el primer caso lo que ocurre es que se vuelve a la capa externa de la que se partió, pero con un conocimiento más estable y seguro acerca de lo que ha hecho o debe hacer. A veces, no sólo se vuelve a la capa de la que se partía, sino que el impulso puede llevar incluso a una capa aún más exterior. En el segundo caso se produce una ruptura no produciéndose un avance en la comprensión.

En este trabajo vamos a centrarnos en los distintos tipos de folding back, que aparecen en la comprensión del concepto de serie numérica, cuando dos alumnos resuelven una tarea planteada y que se describe a continuación.

\section{Metodología}

Se ha analizado la transcripción de la grabación de una sesión de clase de una pareja de alumnos de primer curso de Ingeniería Técnica en Informática de una Universidad privada española mientras resolvía una tarea que involucra el estudio del carácter de dos series numéricas. El trabajo de los estudiantes en clase se grabó mediante una cámara web, se transcribió la grabación de audio y se visualizó el video para completar la información con su lenguaje corporal y con las acciones que llevan a cabo en momentos de silencio, por ejemplo cuando consultan el cuaderno de apuntes.

Las series numéricas se estudiaron en el segundo cuatrimestre de la asignatura Fundamentos Matemáticos I, en el tema de sucesiones y series numéricas. Los conocimientos previos de los estudiantes antes de comenzar el tema son los adquiridos en enseñanza secundaria. En las clases, el concepto de serie numérica se introdujo a partir de una tarea relacionada con la serie geométrica de razón 1/2 (Codes \& Sierra, 2007); se demostró el criterio de convergencia de las series geométricas, el criterio de comparación y el criterio de la integral, entre otros, y este último se utilizó para probar el carácter de las series armónicas $\left(\sum_{n} \frac{1}{n^{\alpha}}\right.$ con $\alpha$ real). Tanto las series geométricas 
como las armónicas se utilizaron en ejercicios rutinarios en los que se podía aplicar el criterio de comparación. Además, la profesora explicó en clase cómo Oresme dedujo la divergencia de la serie $\sum_{n} \frac{1}{n}$ agrupando términos cuya suma es mayor que 1/2.

Los datos que se presentan en esta comunicación provienen de uno de los grupos analizados formado por dos estudiantes con buen expediente académico, no sólo en la asignatura de matemáticas, sino en general en todas las asignaturas. Se seleccionaron estos alumnos porque el análisis realizado reveló una mayor variedad en el mecanismo de folding back. Aunque no eran compañeros habituales de clase, aceptaron que se les grabara mientras resolvían un ejercicio llamado actividad La Torre. Este ejercicio se propuso para que los estudiantes lo resolvieran en clase trabajando en pequeños grupos ( 2 o 3 personas) al final del tema de sucesiones y series numéricas, después de haber definido la suma de una serie numérica como el límite de una sucesión de sumas parciales, y de haber utilizado los criterios de convergencia tanto en ejercicios rutinarios de estudio del carácter de una serie, como en ejercicios en los que la sucesión de sumas parciales se visualiza, principalmente, en un contexto geométrico.

El enunciado que se presentó a los alumnos es el siguiente:

Se quiere construir una torre formada por cilindros de altura igual al radio de la base. El radio de la base es inversamente proporcional a la posición del cilindro en la torre, de modo que el radio del primer cilindro es 1 , el del segundo es $1 / 2$, el del tercero $1 / 3$ y así sucesivamente. Si no hay restricciones en el número de piezas para construir la torre, ¿cuál es la altura y el volumen final de la torre? Deja los resultados indicados.

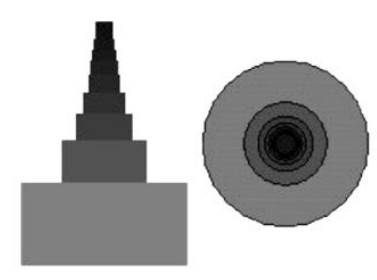

Figura 2. Enunciado de la actividad La Torre.

El contexto del enunciado se corresponde con una situación inviable en la vida real, pero factible en el contexto de un problema matemático en el que los estudiantes han de concluir el volumen y la altura de una torre cuya construcción conlleva un número infinito de pasos. Para resolver el ejercicio los estudiantes deben plantear dos series armónicas, una modeliza la altura y otra el volumen final de la torre, y estudiar su carácter que en el primer caso es divergente y en el segundo convergente.

\section{Análisis y Resultados}

El análisis de la discusión del grupo de alumnos mientras resolvían la tarea anteriormente descrita se ha organizado en tres partes diferenciadas que se corresponden con: el proceso que siguen hasta que determinan las series correspondientes a la altura y al volumen de la torre, la determinación del carácter de la serie de la altura y la del estudio del carácter de la serie correspondiente al volumen de la torre. 


\subsection{Determinación de las Series de la Altura y el Volumen de la Torre}

Los alumnos inicialmente leen con detenimiento el enunciado de la tarea fijándose en los elementos geométricos (radio y altura) de cada cilindro para hacerse una idea inicial de cómo se ha construido la torre. Han de establecer la relación que existe entre la altura y el radio de cada cilindro por lo que comienzan enfrentándose al problema desde el nivel Image Making.

José M. La altura de cada cilindro, ¿cuánto es? Siempre la misma, ¿o no? O sea, la altura de cada cilindro, ¿cuánto es?

Carlos La altura no...

José M. La altura de cada cilindro.

Carlos No sé. Hache. No, no te da un dato.

José M. Sí, pero tiene que tener una altura.

Carlos Bueno, siempre va a ser positiva. O sea, no sé si variará.

Como les surgen algunas dudas para tener una imagen de cómo está construida la torre, precisan la ayuda de la profesora que lee con ellos el enunciado del problema haciendo hincapié en la frase "de altura igual al radio".

José M. Eh... A ver.

Profesora Voy.

José. M. La altura de cada cilindro es una constante o qué...

Carlos ¿O varía?

José M. ¿O varía, o...?

Profesora Sí, varía ¿no? Dice...

José M. El radio no, la altura.

Profesora Sí, sí, sí. Dice: se quiere construir una torre formada por cilindros de altura igual al radio de la base.

José M. ¡Ah! Altura igual al radio de la base.

Con esta pequeña ayuda los alumnos se dan cuenta, casi de repente, de cómo se ha construido la torre lo que les permite acceder a un nivel exterior de comprensión, Image Having, en el que van a ser capaces de actuar sobre sus representaciones mentales, sin necesidad de objetos concretos puesto que ya tienen la imagen de la construcción.

En este momento ya saben cuáles son los datos de los que disponen, cómo están relacionados y pueden escoger la estrategia que les permita resolver la tarea. De hecho en el siguiente diálogo se puede comprobar cómo han interiorizado que la altura y el radio de cada cilindro son iguales. Así, empiezan a organizar los datos para avanzar en el proceso de resolver la tarea.

José M. ¿Cuál es la altura y el volumen final? Pues la altura, la altura va a ser... Mejor se... se saca primero el...

Carlos ¿Cuál es la altura final?

José M. Altura y el volumen. Sacamos la... 
Carlos La altura es sólo como los radios, ¿sabes?

José M. La altura... Pero sacamos primero la expresión general...

Carlos Sí.

José M. ... de lo que es cada cilindro, y después el sumatorio es la altura, ¿no?

Carlos Vamos a sacar la expresión de cómo varían los radios.

José M. Eso. Bueno, los radios que es la altura, claro.

Es en este momento cuándo utilizan un lenguaje simbólico matemático para expresar la altura de un cilindro cualquiera (el que ocupa la posición n-ésima). Ser capaces de expresar una propiedad general de esta índole constituye una evidencia de que han alcanzado el nivel Property Noticing.

Carlos Sí. A uno partido... Sí es ésta, a uno partido de ene.

Finalmente, se desligan por completo de la imagen concreta y consideran el concepto como objeto formal determinando cuál es la serie correspondiente a las alturas (Formalising).

José M. Es igual a uno partido ene. Sí, pero no. Pero desde... desde ene igual a uno.

Una vez determinada la serie correspondiente a la altura han de hacer lo mismo con la serie correspondiente al volumen de la torre. Para ello necesitan hacer algunos cálculos en los que interviene la fórmula del volumen de un cilindro. Como hacen un proceso de recuerdo de esos conocimientos, que no están relacionados con el concepto de serie numérica, esto constituye una muestra de cuándo los alumnos recurren a su Primitive Knowing. En definitiva, en este momento se produce un paréntesis en el que los alumnos no se están planteando la construcción de la serie correspondiente al volumen, simplemente necesitan un recurso concreto para seguir avanzado en la resolución de la tarea y poder expresar la serie correspondiente al volumen de la torre.

José M. Y el volumen, es... Volumen sub ene es... pi al cuadrado por hache. Entonces es pi...

Carlos El radio al cubo.

Esto constituye un folding back puesto que para determinar la serie correspondiente al volumen de la torre, estos alumnos parten de la construcción de la serie de la altura y junto con el recuerdo de la fórmula del volumen del cilindro son capaces de pasar directamente al nivel Property Noticing sin necesidad de progresar por los niveles más interiores. Este tipo de folding back es el que Martín (2008) denomina collecting.

José M. $\quad$... y el radio es uno partido de ene al cubo. Ya está. Ese es el volumen sub ene.

A partir de esta intervención, se produce de forma casi inmediata la generalización y determinación de la expresión correspondiente a la serie del volumen (Formalising).

Carlos $\quad$ o sea, que el volumen total... 
José M. Entonces, el volumen total es el sumatorio desde ene igual a uno de pi...

Carlos $P i$.

José M. ...por pi, eh... uno partido de ene al cubo.

En la siguiente figura se puede ver el proceso que han seguido los alumnos hasta el momento de determinación de las dos series. En el gráfico, se ha representado con una línea discontinua los saltos entre diferentes niveles que realizan los alumnos tras un folding back efectivo.

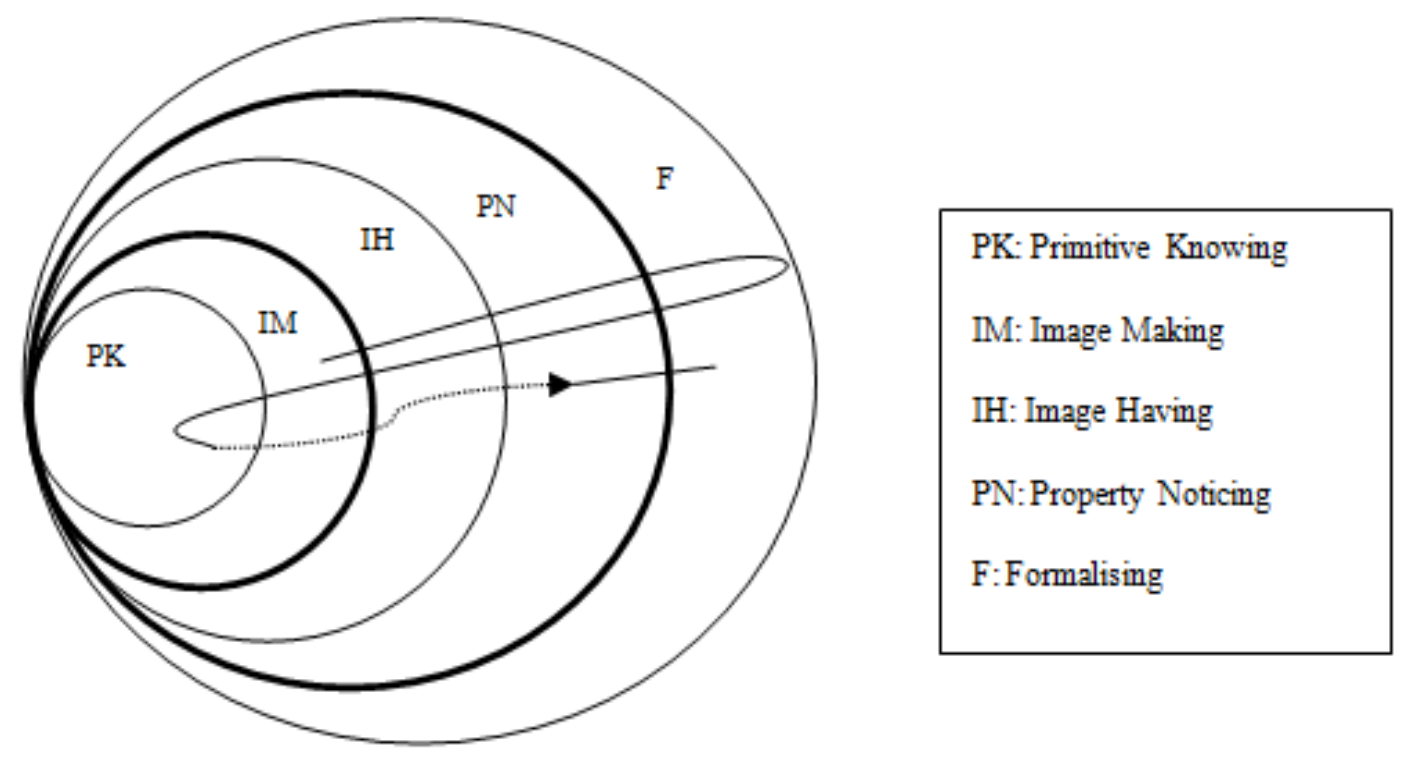

Figura 3. Proceso seguido por los alumnos para determinar las dos series

\subsection{Estudio de la Convergencia de la Serie Correspondiente a la Altura.}

La primera idea que se les ocurre para determinar la convergencia de la serie de la altura de la torre es comprobar si es una serie geométrica ya que anteriormente a esta tarea habían trabajado con este tipo de series (Codes, 2010). Además, están pensando no sólo en determinar si la serie es o no convergente sino calcular la suma de la serie, por ello tratan de establecer cuál es la razón de la serie como si se tratara de una serie geométrica. Demuestran de esta forma que son capaces de utilizar un conocimiento anterior y aplicarlo, lo que constituye de nuevo un folding back to collect (Martin, 2008).

José M. ... ¿Ahora cómo se hace el sumatorio? La razón... [Están buscando en los apuntes] ¿Tienes tú la fórmula del sumatorio?

Comparan las expresiones algebraicas de las series geométricas que tienen en sus apuntes y la expresión de la serie correspondiente a la altura de la torre que han obtenido y se dan cuenta de que su serie empieza desde $\mathrm{n}$ igual a $1 \mathrm{y}$ las geométricas que tienen en sus apuntes desde $\mathrm{n}$ igual a 0 . Comienzan a discutir qué pueden hacer para que su expresión empiece desde 0 y así "arreglar su serie". Pero se encuentran con la dificultad de que si modifican la serie, el primer término tendría como denominador cero. 
Carlos Esto y... pero hay que saber... n está entre cero y uno, sí, siempre, sí.

José M. Bueno, es que siempre esta, siempre empieza en uno, hay que quitar después lo que valga para cero. Pero es que lo que valga para cero no se puede sacar.

Carlos Pues cero. Ah pero para cero...

José M. Para cero, ¿cuánto vale?

Carlos Cero. Es verdad, no podemos dar ese valor. Esta va a ser...

José M. No, ¿sabes lo que podemos hacer?, hacerlo desde cero y que salga la misma. Iba a decir, hacerlo desde cero y cambiar la expresión. Ene menos uno. No al revés es,...

Carlos Sí.

José M. ... ¿Sabes cómo te digo? O sea, en lugar de decir ene igual a uno, dices ese hache igual a sumatorio de [lo está escribiendo mientras lo dice] y ene igual a cero, de uno partido de... Vamos a ver, tenemos un problema, que si lo hacemos con ene igual a uno...

Carlos No podemos restar...

José M. ... no podemos después restar porque el denominador es cero.

Este razonamiento corresponde al nivel Image Making puesto que están intentando adaptar la serie a otra que ellos conocen para luego poder estudiar la convergencia. Al identificar su serie con una geométrica no se están dando cuenta de que están confundiendo en una potencia el exponente con la base (Warner, 2008).

Para superar esta dificultad necesitaron la intervención de la profesora que les ayudó a identificar que no se trataba de una serie geométrica, con lo que alcanzan el nivel Property Noticing. Superan su confusión de base y exponente lo que constituye una reflexión autoconsciente (Martin, 2008) al cuestionarse sobre su propia comprensión acerca de dichos conceptos y reconocer que no los estaban utilizando de forma adecuada y debían reajustarlos para resolver la tarea:

Profesora ¿Cómo es una serie geométrica? … ¿Cómo es?

José M. Lo que va a multiplicar, eh elevado a ene, ¿no?

Profesora Erre, uno partido de... bueno, erre a la ene.

José M. Erre a la ene, sí. Y claro esta no está partido...

De nuevo han de estudiar la expresión que tiene su serie para poder determinar su carácter por lo que vuelven al nivel Image Making. Casi de una forma inmediata se dan cuenta de que la serie de las alturas diverge al recordar que en clase se dedujo la divergencia de la serie armónica utilizando el criterio de la integral y asociando la demostración con Oresme con lo que alcanzan el nivel Property Noticing.

Carlos Esta es infinito. Esta es infinito, la de arriba.

José M. Lo dejamos con uno [estaba rectificado la fórmula del sumatorio].

Carlos No, no. Míralo.

José M. [Está escribiendo] ene, pi partido de ene. ¿Eh?, ah claro, esa es infinito. 
Carlos Esta es seguro infinito.

José M. Esta es igual a infinito por Oresme. Hala, ya está. Por lo que vimos ayer de..., por eso.

Confirman la divergencia de la serie cuando aplican el test de la integral que se había manejado durante las clases teóricas para establecer el carácter de las series armónicas generalizadas. Como están utilizando una propiedad o regla matemática de forma general aplicándola a un objeto matemático se encuentran en el nivel Formalising:

Carlos Miralo. Espérate, pero era... Alfa.

José M. Espérate, que voy...

Carlos Míralo, alfa igual, menor o igual que esto, no converge. Y con la de uno partido de ene también.

José M. Uno partido de..., si alfa es...

Carlos ...igual a uno, no converge.

José M. No converge, con lo cual tiende a infinito, ¿no?

Carlos Hala. Sí, porque a menos infinito es imposible. Tiene que ser positivo.

José M. Claro, por el criterio integral.

En la figura 4 se muestra el proceso que han seguido los alumnos hasta el momento.

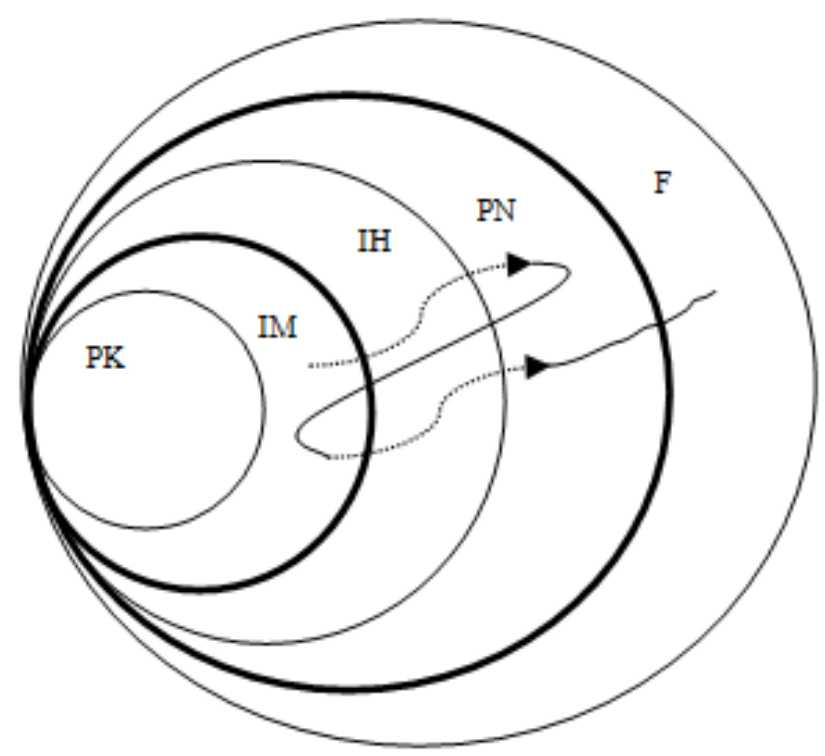

Figura 4. Proceso seguido por los alumnos en el estudio de la convergencia de la serie correspondiente a la altura

\subsection{Estudio de la Convergencia de la Serie Correspondiente al Volumen.}

$\mathrm{Al}$ igual que hicieron al determinar la serie correspondiente al volumen, ahora para estudiar su convergencia utilizan lo que conocen sobre el carácter de la serie de las 
alturas combinándolo con la aplicación del criterio de la integral. Como están aplicando algo que ya han utilizado a una nueva situación, siguen en el nivel Formalising.

José M. Pero, ¿y la otra? Si la otra es igual.

Carlos No, porque tiene pi. Por el criterio integral no. Míralo. Es uno partido ene elevado a...

José M. Si alfa es mayor que uno... converge.

Carlos Mayor que uno, converge. Pero esto es uno. Bueno, converge, sí, a ... por pi.

José M. Aunque sea pi, converge.

Carlos Pero no sabemos a qué. Y además esto no es pi, es.... Y aqui es pi.

José M. Yo creo que eso no te...

Carlos Yaquí es pi.

José M. Ya, y que ese uno es....

Carlos Que es distinto.

Esta última intervención de Carlos les lleva a un folding back (Pirie y Kieren, 1992). Al aparecer el número $\pi$ en la expresión simbólica de la serie se muestran inseguros de que se pueda aplicar el mismo criterio que antes. La dificultad que han encontrado es de índole algebraica, no está tan asociada con el establecimiento del carácter de la serie cuanto con la aplicación de la propiedad de la linealidad: si una serie converge, al multiplicarla por un número sigue convergiendo. Aquí, en realidad tienen que aplicar esta propiedad en sentido inverso, es decir, determinar que la serie del volumen converge porque esa serie sin el factor $\pi$ converge.

La inseguridad de Carlos les conduce a revisar otros criterios de convergencia de series de números reales de términos positivos para resolver este problema. Esto implica comenzar a aplicar cada criterio desde el nivel Image Making, iniciando este proceso con el criterio de sándwich:

José M. Venga vamos a ver, un momento. Si, si esa... mira por lo del sándwich. Si es pi, aquella va a estar por encima. Si esa converge, ah, esa no sabemos si converge.

Carlos Va a estar por encima.

José M. Y esa converge, entonces no sabemos si converge o no converge. No te fastidia. Si estuviera por debajo sí, tendría que converger.

Como no llegan a ninguna conclusión valoran calcular el límite del término nésimo aunque esa sólo es una condición necesaria pero no suficiente para determinar la convergencia de la serie.

Carlos El término n-ésimo no nos dice nada, porque ya lo he mirado yo.

José M. ¿Has mirado lo del término n-ésimo, tú?

Carlos Míralo, siempre va a ser más grande, tiende a cero. O sea, que no se sabe nada.

José M. Siempre va a tender a cero. 
Carlos No se sabe nada.

José M. Si no tendiera a cero.

Intentan aplicar el criterio de comparación buscando una serie que sea mayor que la que tienen y que converja:

José M.: Criterio de comparación. ¿No tienes tú estos que hicimos... que eran así estándares?

Carlos: ¿Cuál?

José M.: Por comparar. Uno que sea mayor que este, que converja. ¿Sabes? Mayor que éste y que converja. ¿Cuál podría ser? Cualquier exponencial es mayor que este. No, cualquier exponencial es más pequeño, porque está dividiendo. Claro, ¿o no? ... una exponencial.

Carlos Más pequeño.

José M. [Susurrando] uno partido de... Por ejemplo, uno partido de ene al cuadrado es más pequeña, ¿no? Ah no, pi.

Carlos ¿Uno partido...?

José M. De ene al cuadrado. Es más pequeña.

Carlos Al principio, pero luego... Más pequeño al principio, los primeros términos.

Como no consiguen encontrar una serie que les permita aplicar el criterio anterior, lo intentan con el criterio del logaritmo:

José M. Vamos a ver con el criterio del logaritmo. (...) Converge. Ya está, ya lo tengo, converge. ¿Sabes? Lo he hecho con éste, con el criterio del logaritmo. A ver, es... el logaritmo neperiano de uno partido de pi elevado a... partido de ene al cubo, ¿no? ¿A ver cómo sería? [Escribe].

Carlos $\quad S i ́$

José M. Partido del logaritmo neperiano de ene.

Carlos Sí.

José M. Es decir, logaritmo neperiano de ene es ene al cubo partido de pi, ¿no? Y logaritmo neperiano de ene. Logaritmo neperiano de ene tiende a infinito, ¿no? Y esto te... bueno, lo sacas y dices tres por el logaritmo neperiano de ene, que tiende a infinito, menos $k$, eh no, logaritmo de en ... logaritmo neperiano de pi, que es un número $K$, ¿no? Entonces dices, como este es tres y este tiendo a infinito, logaritmo neperiano de ene igual que aquí, bueno hago este logaritmo, ¿no? Pues tres partido... tres, converge. Es mayor que... es mayor ... converge. Lambda es mayor que uno, converge.

En la siguiente figura se ilustra el proceso seguido por los alumnos en este apartado. 


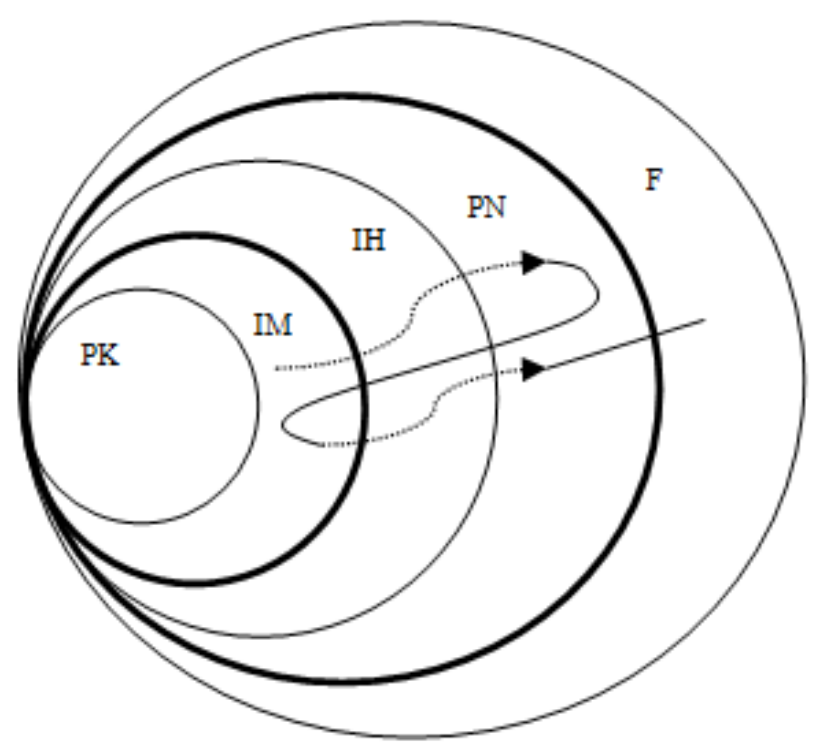

Figura 5. Proceso seguido por los alumnos en el estudio de la convergencia de la serie correspondiente al volumen.

Aunque no han reconocido la serie como una armónica generalizada sí han sido capaces de establecer el carácter de la serie mediante el criterio del logaritmo por lo que llegan al nivel Property Noticing.

\section{Conclusiones}

El modelo de Pirie-Kieren permite analizar el proceso de comprensión del conocimiento de una pareja de alumnos cuando interactúan entre ellos. Esta construcción, tal y como se ha visto en el estudio presentado, se plantea como un proceso colectivo, en el que las interacciones entre iguales y con el profesor juegan un papel fundamental.

Si algo demuestra el modelo de Pirie-Kieren es que la comprensión del conocimiento no se produce de forma lineal, ni siempre aparece como un avance. En muchas ocasiones, y de forma natural, es necesaria una "vuelta atrás" en el proceso, lo que este modelo denomina folding back, para buscar una información necesaria o para reforzar un conocimiento que no está suficientemente consolidado. El mecanismo folding back forma parte esencial de la comprensión, y se convierte en un elemento necesario para fortalecer el crecimiento de la comprensión, para hacerlo más consistente y más estable de cara a un avance posterior.

En las intervenciones aquí presentadas se evidencia el recurso al mecanismo folding back en tres ocasiones. En el primer caso, cuando construyen las dos series de la altura y el volumen, se les plantea una duda de geometría elemental, que les lleva desde el nivel Formalising al nivel Primitive Knowing en busca de ese contenido concreto. Martín (2008) llama collecting a este folding back que se produce cuando se vuelve a un nivel interior para recuperar un conocimiento $\mathrm{y}$, una vez recogido, se regresa al punto en el que estaban. Los alumnos no tenían ninguna duda sobre la construcción, sólo necesitaban una "pieza” más, y sabían dónde buscarla. 
El segundo folding back aparece en el estudio de la convergencia de la serie de la altura. A partir del nivel Property Noticing al que han llegado mediante una reflexión autoconsciente que les permite superar sus confusiones iniciales sobre la naturaleza de la serie y reajustar los conceptos para resolver la tarea, vuelven al nivel Image Making, para estudiar el tipo de expresión de la serie y determinar su carácter. Gracias a este folding back to collect (Martin, 2008) los alumnos recuperan los conocimientos adquiridos sobre criterios de convergencia específicos trabajados previamente en clase y avanzan directamente al nivel Formalising.

El tercer folding back ocurre por un obstáculo que tienen los alumnos cuando han de manejar la expresión algebraica de las series. En este caso el folding back es de naturaleza distinta puesto que se refiere a la falta de manejo de la propiedad de la linealidad de una serie. El obstáculo que produce este folding back responde al principio de extensión genérica (Tall, 1991) según el cual trabajar con ejemplos que comparten cierta propiedad conlleva que los estudiantes tiendan a asumir esa propiedad en otros contextos provocando obstáculos. En este caso, el posible abuso por parte de la profesora de ejemplos en los que la expresión del término general de una serie no contiene ningún factor constante, promueve que los estudiantes no recurran a las propiedades aritméticas de las series para emplear el resultado "si $\sum a_{n}$ es una serie de términos positivos, entonces $\sum k \cdot a_{n}=k \cdot \sum a_{n}$, siendo $k$ constante". De ahí surge la necesidad de comprobar la convergencia de la serie mediante otros criterios que finalmente logran con el criterio del logaritmo.

La realización de la tarea por parte de estos alumnos ha estado caracterizada por el aprovechamiento de los conocimientos previos que poseían y, porque a partir de las construcciones intermedias que han surgido en la resolución de la tarea, han ido progresando entre los niveles de Pirie y Kieren. Esta segunda característica no se ha observado en otro grupo de alumnos analizado (Codes, Gonzalez, et al., 2013).

Según la clasificación de Martin (2008), los tres folding back observados en este estudio surgieron de los estudiantes y fueron deliberados, a diferencia de los observados en Codes, Gonzalez, et al. (2013), donde la intervención del profesor fue uno de los factores externos que provocaron el folding back.

\section{Referencias bibliográficas}

Carlsen, M. (2010). Appropriating geometric series as a cultural tool: a study of student collaborative Learning. Educational Studies in Mathematics, 74, 95-116. doi: 10.1007/s10649-010-9230-0.

Codes, M. (2010). Análisis de la comprensión de los conceptos de serie numérica y su convergencia en estudiantes de primer curso de universidad utilizando un entorno computacional. (Tesis doctoral). Universidad de Salamanca, Salamanca, España. http://hdl.handle.net/10366/76452

Codes, M., Delgado, M. L., González Astudillo, M. T., \& Monterrubio, M. C. (2013) Comprensión del concepto de serie numérica a través del modelo de Pirie y Kieren. Enseñanza de las Ciencias, 31(3), 135-154.

Codes, M., González, M.T., Delgado, M.L., \& Monterrubio, M.C. (2013). Growth in the understanding of the concept of infinite numerical series: a glance through Pirie and Kieren theory. International Journal of Mathematical Education in Science and Technology, 44(5), 652-662. 
Codes, M., \& Sierra M. (2007). Actividad Rectángulos: Un ejemplo de aplicación de metodologías activas en el aula universitaria de matemáticas. Actas de las IV Jornadas internacionales de Innovación Universitaria (2007). Madrid: Universidad Europea de Madrid.

Delgado, M. L., González, M. T., Monterrubio, C., \& Codes, M. (2013). El mecanismo collecting para la comprensión del concepto de serie numérica. En A. Berciano, G. Gutiérrez, A. Estepa \& N. Climent (Eds.), Investigación en Educación Matemática XVII (pp. 245-252). Bilbao: SEIEM.

Dubinsky, E., Weller, K., McDonald, M. A., \& Brown, A. (2005). Some historical issues and paradoxes regarding the concept of infinity: an APOS-based analysis: part II. Educational Studies in Mathematics, 60, 253-266.

González-Martín, A. S., Nardi, E., \& Biza, I. (2011). Conceptually-driven and visually-rich tasks in texts and teaching practice: the case of infinite series. International Journal of Mathematical Education in Science and Technology, 42(5), 565-589.

Kidron, I. (2002). Concept definition, concept image, and the notion of infinite sum in old and new environments. En A. D. Cockbrun \& E. Nardi (Eds.), 26th International Conference for the Psychology of Mathematics Education, 3, 209216.

McDonald, M. A., Mathews, D. M., \& Strobel, K. H. (2000). Understanding sequences: A tale of two objects, En J. Kaput, A. H. Schoenfeld, \& E. Dubinsky (Eds.), Research in collegiate mathematics education. IV. Conference Board of the Mathematical Sciences (CBMS), Issues in Mathematics Education, 8, 77-102

Martin, L. C. (2008). Folding back and the dynamical growth of mathematical understanding: Elaborating the Pirie-Kieren Theory. The Journal of Mathematical Behavior, 27, 64-85.

Martínez-Planell, R., Gonzalez, A. C., DiCristina, G., \& Acevedo, V. (2012). Students' conception of infinite series. Educational Studies in Mathematics, 81, 235-249.

Pirie, S., \& Kieren, T. (1992). Creating constructivist environments and constructing creative mathematics. Educational Studies in Mathematics, 23, 505-528.

Pirie, S., \& Kieren, T. (1994). Growth in mathematical understanding: How can we characterize it and how can we represent it? Educational Studies in Mathematics, 26, 165-190.

Pirie, S., \& Martin, L. C. (2000). The role of collecting in the growth of mathematical understanding. Mathematics Education Research Journal, 12(2), 127-146.

Tall, D. (1991). The psychology of Advanced Mathematical Thinking. En D. Tall (Ed.), Advanced Mathematical Thinking (pp. 3-21). Dordrecht: Kluwer Academic Publishers.

Warner, L. B. (2008). How do students' behaviors relate to the growth of their mathematical ideas? Journal of Mathematical Behavior, 27, 206-227.

Weller, K., Brown, A., Dubinsky, E., McDonald, M., \& Stenger, C. (2004). Intimations of infinity. Notices of the American Mathematical Society, 51(7), 741750 . 
Delgado, M. L., Codes, M., Monterrubio, M. C., \& González Astudillo, M. T.

\section{Referencias a los autores}

M. Laura Delgado Martín, Universidad de Salamanca (España). laura@usal.es

Myriam Codes Valcarce, Universidad de Salamanca (España). mcodes@usal.es

M. Consuelo Monterrubio Pérez, Universidad de Salamanca (España).

chelomonterrubio@usal.es

M. Teresa González Astudillo, Universidad de Salamanca (España). maite@usal.es 


\title{
The concept of numerical series. A study with Pirie and Kieren model focused on "folding back" mechanism
}

\author{
M. Laura Delgado Martín, Universidad de Salamanca (España) \\ Myriam Codes Valcarce, Universidad de Salamanca (España) \\ M. Consuelo Monterrubio Pérez, Universidad de Salamanca (España) \\ M. Teresa González Astudillo, Universidad de Salamanca (España)
}

\begin{abstract}
In this paper, we present a research about the process followed by a group of university students to build numerical series and to determine its convergence. The aim of this study is describe the process followed by a group of university students to build the numerical series concept while they are solving a task in the usual classroom.

The activity of the students is analysed through the Pirie and Kieren model. This model identifies different layers that are represented by eight nested circles describing the personal growth of understanding about a specific topic. This growth is not linear and each layer contains the previous one, which in turn is contained in the following one, thus representing a continuous forth and back movement that maintains the characteristics from the lower levels that has passed through. These layers are Primitive Knowing, Image-Making, Image-Having, Property Noticing, Formalising, Observing, Structuring and Inventising.

It has been observed that a qualitative and quantitative change in the understanding of a concept can be produced by a mechanism known under the term "folding back", that is, a necessary going back to an inner level that can be caused by different reasons. From a mathematical point of view, individuals use apparently less advanced ways of knowing concepts to produce a better understanding. This mechanism has four different forms: when the student has difficulties with a task he goes back to an inner lever to work in a less sophisticated way in which he is more confident, when a student recover some knowledge already built, when he must return to Primitive Knowledge level to look for other knowledge needed to solve the task and when he has not connected the primitive conceptions with the new one.

In this paper, we report on an activity about numerical series that was presented to a group of students working in a collaborative environment during a fifty-minute session. They are first year students registered in the subject Fundamentos Matemáticos I (Basic Mathematics I) of a Systems Engineering Degree in a Spanish University.

To solve the task the students use the folding back mechanism three times. Firstly, when they have a doubt about a geometrical concept they return from Formalizing level to Primitive Knowing level to find out the piece they need. Secondly, they recover the knowledge they have about convergence criteria explained during the usual class. Finally when they must confront an obstacle about the linearity property of numerical series.
\end{abstract}

